\title{
Search for an extended VHE $\gamma$-ray emission from Mrk 421 and Mrk 501 with the MAGIC Telescope
}

J. Aleksić ${ }^{1}$, L. A. Antonelli ${ }^{2}$, P. Antoranz ${ }^{3}$, M. Backes ${ }^{4}$, C. Baixeras ${ }^{5}$, J. A. Barrio ${ }^{6}$, D. Bastieri ${ }^{7}$, J. Becerra González ${ }^{8}$, W. Bednarek ${ }^{9}$, A. Berdyugin ${ }^{10}$, K. Berger ${ }^{10}$, E. Bernardini ${ }^{11}$, A. Biland ${ }^{12}$, O. Blanch ${ }^{1}$, R. K. Bock ${ }^{13,7}$, G. Bonnoli ${ }^{2}$, P. Bordas ${ }^{14}$, D. Borla Tridon ${ }^{13}$, V. Bosch-Ramon ${ }^{14}$, D. Bose ${ }^{6}$, I. Braun ${ }^{12}$, T. Bretz $^{15}$, D. Britzger ${ }^{13}$, M. Camara ${ }^{6}$, E. Carmona ${ }^{13}$, A. Carosi ${ }^{2}$, P. Colin ${ }^{13}$, S. Commichau ${ }^{12}$, J. L. Contreras ${ }^{6}$, J. Cortina ${ }^{1}$, M. T. Costado ${ }^{8,16}$, S. Covino ${ }^{2}$, F. Dazzi $1^{17}, \star$, A. De Angelis ${ }^{17}$, E. De Cea del Pozo ${ }^{18}$, R. De los Reyes ${ }^{6, \star \star}$, B. De Lotto ${ }^{17}$, M. De Maria ${ }^{17}$, F. De Sabata $^{17}$, C. Delgado Mendez ${ }^{8, \star \star}$, M. Doert ${ }^{4}$, A. Domínguez ${ }^{19}$, D. Dominis Prester ${ }^{20}$, D. Dorner ${ }^{12}$, M. Doro ${ }^{7}$, D. Elsaesser ${ }^{15}$, M. Errando ${ }^{1}$, D. Ferenc ${ }^{20}$, M. V. Fonseca ${ }^{6}$, L. Font ${ }^{5}$, R. J. García López ${ }^{8,16}$, M. Garczarczyk ${ }^{8}$, M. Gaug ${ }^{8}$, N. Godinovic ${ }^{20}$, D. Hadasch ${ }^{18}$, A. Herrero ${ }^{8,16}$, D. Hildebrand ${ }^{12}$, D. Höhne-Mönch ${ }^{15}$, J. Hose ${ }^{13}$, D. Hrupec ${ }^{20}$, C. C. $\mathrm{Hsu}^{13}$, T. Jogler ${ }^{13}$, S. Klepser ${ }^{1}$, T. Krähenbühl ${ }^{12}$, D. Kranich ${ }^{12}$, A. La Barbera ${ }^{2}$, A. Laille ${ }^{21}$, E. Leonardo ${ }^{3}$, E. Lindfors ${ }^{10}$, S. Lombardi ${ }^{7}$, F. Longo ${ }^{17}$, M. López $^{7}$, E. Lorenz ${ }^{12,13}$, P. Majumdar ${ }^{11}$, G. Maneva ${ }^{22}$, N. Mankuzhiyil ${ }^{17}$, K. Mannheim ${ }^{15}$, L. Maraschi ${ }^{2}$, M. Mariotti ${ }^{7}$, M. Martínez ${ }^{1}$, D. Mazin ${ }^{1}$, M. Meucci ${ }^{3}$, J. M. Miranda ${ }^{3}$, R. Mirzoyan ${ }^{13, \dagger}$, H. Miyamoto ${ }^{13}$, J. Moldón ${ }^{14}$, M. Moles ${ }^{19}$, A. Moralejo ${ }^{1}$, D. Nieto ${ }^{6}$, K. Nilsson ${ }^{10}$, J. Ninkovic ${ }^{13}$, R. Orito ${ }^{13}$, I. Oya ${ }^{6}$, S. Paiano ${ }^{7}$, R. Paoletti ${ }^{3}$, J. M. Paredes ${ }^{14}$, S. Partini ${ }^{3}$, M. Pasanen ${ }^{10}$, D. Pascoli ${ }^{7}$, F. Pauss ${ }^{12}$, R. G. Pegna ${ }^{3}$, M. A. Perez-Torres ${ }^{19}$, M. Persic ${ }^{17,23}$, L. Peruzzo ${ }^{7}$, F. Prada ${ }^{19}$, E. Prandini ${ }^{7}$, N. Puchades ${ }^{1}$, I. Puljak ${ }^{20}$, I. Reichardt ${ }^{1}$, W. Rhode ${ }^{4}$, M. Ribó ${ }^{14}$, J. Rico ${ }^{24,1}$, M. Rissi ${ }^{12}$, S. Rügamer ${ }^{15}$, A. Saggion ${ }^{7}$, T. Y. Saito ${ }^{13}$,

M. Salvati ${ }^{2}$, M. Sánchez-Conde ${ }^{19}$, K. Satalecka ${ }^{11}$, V. Scalzotto ${ }^{7}$, V. Scapin ${ }^{17}$, C. Schultz ${ }^{7}$, T. Schweizer ${ }^{13}$,

M. Shayduk ${ }^{13}$, S. N. Shore ${ }^{25}$, A. Sierpowska-Bartosik ${ }^{9}$, A. Sillanpää ${ }^{10}$, J. Sitarek ${ }^{13,9, \ddagger}$, D. Sobczynska ${ }^{9}$, F. Spanier $^{15}$, S. Spiro' ${ }^{2}$, A. Stamerra ${ }^{3}$, B. Steinke ${ }^{13}$, J. C. Struebig ${ }^{15}$, T. Suric ${ }^{20}$, L. Takalo ${ }^{10}$, F. Tavecchio ${ }^{2}$, P. Temnikov ${ }^{22}$, T. Terzic ${ }^{20}$, D. Tescaro ${ }^{1}$, M. Teshima ${ }^{13}$, D. F. Torres ${ }^{24,18}$, H. Vankov $^{22}$, R. M. Wagner ${ }^{13}$, Q. Weitzel ${ }^{12}$, V. Zabalza ${ }^{14}$, F. Zandanel ${ }^{19}$, R. Zanin ${ }^{1}$, A. Neronov ${ }^{26}$, and D. V. Semikoz ${ }^{27}$

(Affiliations can be found after the references)

Received 7 April 2010 / Accepted 12 July 2010

\section{ABSTRACT}

Context. Part of the very high energy $\gamma$-ray radiation coming from extragalactic sources is absorbed through the pair production process on the extragalactic background light photons. Extragalactic magnetic fields alter the trajectories of these cascade pairs and, in turn, convert cosmic background photons to $\gamma$-ray energies by inverse Compton scattering. These secondary photons can form an extended halo around bright VHE sources. Aims. We searched for an extended emission around the bright blazars Mrk 421 and Mrk 501 using the MAGIC telescope data.

Methods. If extended emission is present, the angular distribution of reconstructed $\gamma$-ray arrival directions around the source is broader than for a point-like source. In the analysis of a few tens of hours of observational data taken from Mrk 421 and Mrk 501 we used a newly developed method that provides better angular resolution. This method is based on the usage of multidimensional decision trees. Comparing the measured shapes of angular distributions with those expected from a point-like source one can detect or constrain possible extended emission around the source. We also studied the influence of different types of systematic errors on the shape of the distribution of reconstructed $\gamma$-ray arrival directions for a point source.

Results. We present upper limits for an extended emission calculated for both sources for various source extensions and emission profiles. We obtain upper limits on the extended emission around the Mrk 421 (Mrk 501) on the level of $<5 \%(<4 \%)$ of the Crab Nebula flux above the energy threshold of $300 \mathrm{GeV}$. Using these results we discuss possible constraints on the extragalactic magnetic fields strength around a few times $10^{-15} \mathrm{G}$.

Key words. galaxies: active $-\gamma$-rays: galaxies

\section{Introduction}

\footnotetext{
^ Supported by INFN Padova.

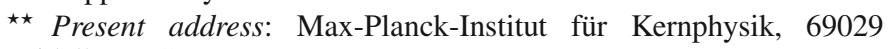
Heidelberg, Germany.

$\star \star \star$ Present address: Centro de Investigaciones Energéticas, Medioambientales y Tecnológicas (CIEMAT), Madrid, Spain.

$\dagger$ Corresponding author: R. Mirzoyan,

e-mail: razmik@mppmu.mpg.de

* Corresponding author: J. Sitarek,

e-mail: jsitarek@mppmu.mpg.de
}

Blazars are well-known extragalactic sources of very high energy (VHE) $\gamma$-rays. These photons traverse large distances through extragalactic space and, therefore, interact with CMB (cosmic microwave background) and EBL (Extragalactic Background Light) photons. As a result, they can be absorbed via the pair production process. This effect leads to a change in the spectral shape of observed spectrum and it can be used to 
constrain the energy density of the EBL (see e.g. Stecker et al. 1992; Mazin et al. 2007).

Possible extended emission around extragalactic sources of VHE $\gamma$-rays was first discussed by Aharonian et al. (1994). $\gamma$-rays with energies greater than $10 \mathrm{TeV}$ are strongly absorbed via a pair production process on EBL and CMB photons relatively close to the source. Secondary $\gamma$-rays can be produced in a cascade initiated by those primary photons. Note that the redshift dependent energy for which the $\gamma$-ray optical depth is about unity varies by a factor of $\sim 3$ between different EBL models (Kneiske et al. 2004; Stecker et al. 2006; Franceschini et al. 2008; Gilmore et al. 2008; Primack et al. 2009; Finke et al. 2010).

Magnetic fields are very non-uniform and their strength can vary within many orders of magnitude depending on their location within the large scale structure. They have been measured thus far only in the galaxies with strength $\sim \mu \mathrm{G}$ (Kulsrud \& Zweibel 2008; Beck 2008), in cores of galaxy clusters (within the inner $100 \mathrm{kpc}) \sim \operatorname{several} \mu \mathrm{G}$ (Carilli \& Taylor 2002), and near the borders of few clusters with strength $10^{-8}-10^{-7} \mathrm{G}$ (on Mpc scales) (Xu et al. 2006; Kronberg et al. 2007). On larger distance scales the magnetic field strengths are not known but they have to be much weaker (see e.g. de Angelis et al. 2008). Moreover, theoretical models predict very weak $\left(B \ll 10^{-12} \mathrm{G}\right)$ extragalactic magnetic fields (EGMF) within voids in large scale structures, outside galaxies and galaxy clusters (Kronberg 1994; Grasso \& Rubinstein 2001; Widrow 2002; Neronov \& Semikoz 2009). It is believed that voids make up a significant part of the space volume.

Development of a secondary $\gamma$-ray cascade depends on its original energy and the strength of surrounding magnetic field. Two cases can be considered. First, if the TeV source is located in a region of intense magnetic field and the maximum $\gamma$-ray energy is high $\left(E_{\max } \gg 50 \mathrm{TeV}\right), \mathrm{e}^{+} \mathrm{e}^{-}$pairs are directly produced by $\gamma$-rays near the source (within a few Mpc). These will be isotropized in any strong magnetic field that may exist around the source host galaxy or galaxy cluster (Aharonian et al. 1994). Second, the mean free path for $\gamma$-rays of moderate energy $(E<50 \mathrm{TeV})$ is longer so they can traverse larger distances from the source. Therefore, $\mathrm{e}^{+} \mathrm{e}^{-}$pairs will be produced in regions with much weaker magnetic fields (Plaga 1995; Neronov \& Semikoz 2007; Elyiv et al. 2009; Dolag et al. 2009). In this case the deflections of the pair trajectories by EGMF are not large enough to isotropize the secondary cascade emission. Instead the inverse Compton scattering of CMB photons by $\mathrm{e}^{+} \mathrm{e}^{-}$ pairs will produce secondary $\gamma$-rays with a slightly different direction than the primary photons. The redirection of the cascade photons into the field of view of the telescope can produce an extended emission region around the point-like source, even when $B \leq 10^{-12} \mathrm{G}$ (Neronov \& Semikoz 2007; Elyiv et al. 2009; Dolag et al. 2009). This extended emission is produced between the source and the observer. For a distant observer this extended emission will be superimposed on the point source, thus mimicking a halo. The secondary $\gamma$-rays can also initiate cascades, provided the optical depth in the EBL radiation field is still large enough but the energies of further generation of $\gamma$-rays may be below the energy threshold of VHE $\gamma$-ray instruments.

Blazars are known to be strongly variable, in particular at $\gamma$-ray energies (e.g. Aharonian et al. 2007; Albert et al. 2008a). The extended emission component cannot follow the original time profile of the emission. This is because the secondary cascade photons do not propagate along the same path as the direct $\gamma$-rays from the primary source. Instead, its variations will be delayed and stretched on much longer time scales (up to $\sim 10^{6}$ years at $\sim 100 \mathrm{GeV}$ energy for $B \sim 10^{-12} \mathrm{G}$,
Neronov \& Semikoz (2007, 2009), see also Plaga 1995; Dai et al. 2002; Murase et al. 2008). Thus the direct emission from the point source will be overlayed on the extended emission component. The latter can constitute a part of the quasi-constant, quiescent emission.

The first attempt to detect extended emission around extragalactic TeV $\gamma$-ray sources was performed by the HEGRA instrument for Mrk 501. It yielded only an upper limit of 5-10\% of the Crab Nebula flux (at energy $\geq 1 \mathrm{TeV}$ ) on angular scales of 0.5 to $1^{\circ}$, (Aharonian et al. 2001a). In this paper we report on our search for extended emission of VHE $\gamma$-rays from the bright blazars Mrk 421 and Mrk 501 using the $17 \mathrm{~m}$ diameter MAGIC imaging atmospheric cherenkov telescope (IACT). The details of the telescope, its performance and the standard analysis chain are described in Albert et al. (2008d). Due to the large size of the mirror dish and improved light sensors, the MAGIC trigger threshold $(\sim 50 \mathrm{GeV})$ is $\sim 2-3$ times lower compared to other operating IACTs. In Sect. 2. we describe the analysis method, which was used for searching for an extended emission. Also, we present a new method that improves the angular resolution. In Sect. 3. we present analyses of possible systematic effects which can mimic the existence of an extended emission. In Sect. 4. we describe the data sample used for the analysis. In Sect. 5. we present the results of the analysis: the upper limits on the extended emission from Mrk 421 and Mrk 501. Finally, in Sect. 6. we discuss how these upper limits constrain the strength of the EGMF.

\section{Analysis method}

We parameterize the images of air showers using the so-called Hillas parameters (Hillas 1985). The angular distance between the center of gravity of the image and the shower direction (designated DISP) is correlated with geometrical and timing properties of the image. The arrival direction for every event can be estimated. The distribution of the squared angular distance between the estimated and true source positions $\left(\theta^{2}\right)$ is narrow and has a peak at $\theta=0$ for a point-like $\gamma$-ray source. For an extended source this distribution will be broader. The DISP parameter is proportional to the ellipticity $(1-$ Width/Length $)$ of the image. By including the dependence on the possible truncation of the image at the edge of the camera and on the parameter Size (sum of total charge of an image), one can improve the precision of DISP (see Domingo-Santamaria et al. 2005).

\subsection{The novel random forest DISP method}

For this study we developed a new method for the DISP estimation that includes the Leakage, the Time Gradient, and the dependence on the zenith angle of observations along with standard Hillas parameters (Width, Length, Size). Leakage is a measure of the truncation of images due to the camera edge effects. It is defined as the ratio of the charge in the last two rings of pixels in the camera to the total charge. The parameter Time Gradient is defined as the derivative of signal arrival time in pixels along the main axis of the image. For a given event, the Time Gradient is strongly correlated with the impact parameter of the parent shower. Since for a given zenith angle and $\gamma$-ray energy the DISP is a simple function of the impact parameter, one can improve the DISP estimation, and as a result also the angular resolution, by using the fast timing properties of the image. The MAGIC telescope's ultra-fast time response because of its parabolic reflector shape, special PMTs, and 2 GSample/s FADC readout enabled us to include the Time Gradient parameter in our analysis, thus enhancing the sensitivity of the telescope (Aliu et al. 2009). 
J. Aleksić et al.: Search for an extended VHE $\gamma$-ray emission from Mrk 421 and Mrk 501 with the MAGIC Telescope

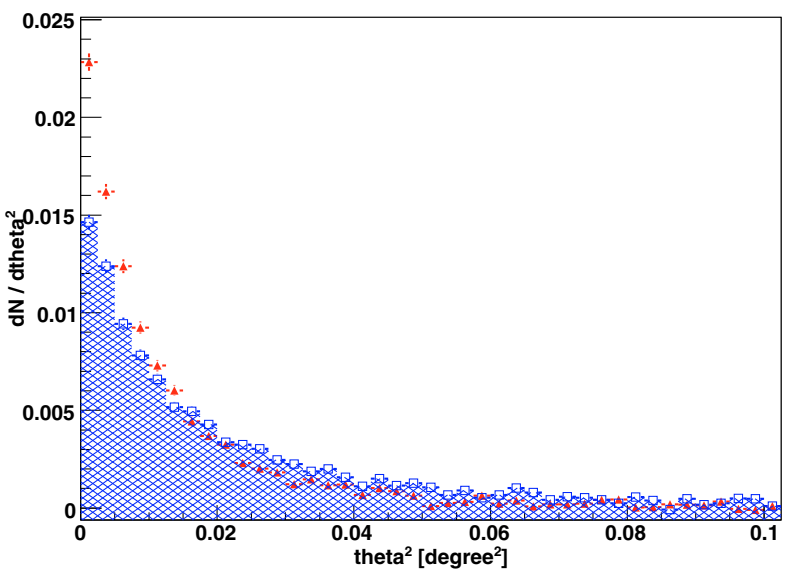

Fig. 1. Comparison between the $\theta^{2}$ distributions of the $\gamma$-ray excess obtained from $43 \mathrm{~h}$ of Crab Nebula data with the parametrized DISP value (blue squares, shaded area), and the RF DISP method (red triangles). (This figure is available in color in electronic form).

To combine information from both the geometrical and timing properties of images in the most efficient way, we used multidimensional decision trees, called the Random Forest (RF) method. It is widely used for the $\gamma /$ hadron separation and the energy estimation (Bock et al. 2004; Albert et al. 2008c). A comparison between the novel RF method (hereafter RF DISP) and the standard parametrized DISP is presented in Fig. 1.

The RF DISP provides a substantially narrower $\theta^{2}$ distribution and improves the angular resolution (defined as the $40 \%$ containment radius for a point-like source, equivalent to one standard deviation of a two dimensional gaussian distribution), by $\sim 20-30 \%$. This improvement is due to using a Random forest method instead of simple parameterization and Time Gradient information. This enhances the telescope performance for the search for an extended emission.

The shape and the width of a $\theta^{2}$ distribution for a point-like source depends on many factors, among which is the energy of the showers. For a higher energy shower, due to the large number of particles in the shower maximum, one has a higher signal-tonoise ratio, and the resulting image has more precisely defined parameters. Thus, we have selected events with large Size (the total measured charge of the image), which improves the precision of the reconstruction of the shower direction. In our analysis we used only showers with $S$ ize $>400$ photoelectrons which allows us to determine the $\theta^{2}$ with relatively high precision. This leads to an energy threshold (defined as the peak of the Monte Carlo simulated differential energy distribution) of $300 \mathrm{GeV}$.

Let us consider a situation where the excess observed from a hypothetical source is a mixture of a point source and a weak extended emission with a given profile. The cumulative $\theta^{2}$ distributions for point-like and extended sources are shown in Fig. 2. Using this figure, the angular resolution for this analysis is estimated to be $\lesssim 0.1^{\circ}$.

\subsection{Source extension}

If the characteristic extension $\theta_{\text {ext }}$ is much smaller than the telescope's point spread function (PSF), the distributions of a purely point-like and a partially extended source are very similar. For larger extensions, clear differences between the distribution shapes will be seen (see Fig. 2). To investigate possible extended emission from blazars we adopted a method similar to that used in Aharonian et al. (2001a). We calculated the ratio of event rates in two $\theta$ ranges: $f=\frac{N\left(\theta_{1}<\theta<\theta_{2}\right)}{N\left(0<\theta<\theta_{1}\right)}$, and compared them with the ratio
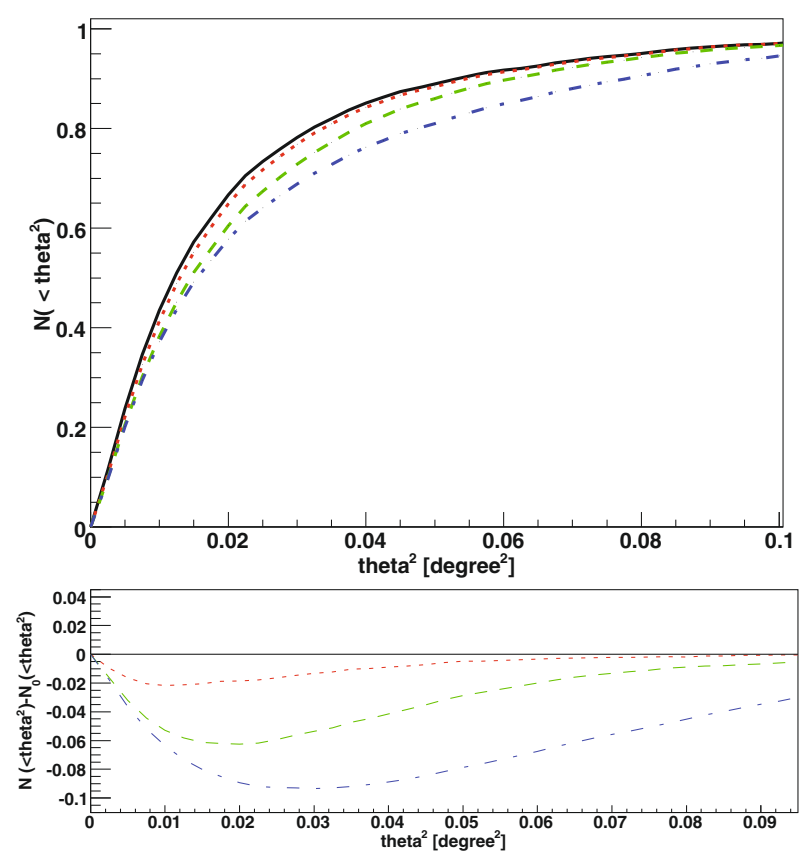

Fig. 2. Comparison of Monte Carlo cumulative $\theta^{2}$ distributions (upper panel) for a source with $80 \%$ point-like and $20 \%$ extended emission and difference between them (lower panel). The characteristic radius of the extension is equal to $0.1^{\circ}$ (red dotted), $0.2^{\circ}$ (green dashed) or $0.3^{\circ}$ (blue dot-dashed), respectively. The extended part of the emission is simulated as a flat disc ( $\mathrm{d} N / \mathrm{d} \theta^{2}=$ const.). The purely point-like source is shown as a black solid line. A random mispointing up to $0.03^{\circ}$ has been included in the simulations. (This figure is available in color in electronic form).

predicted for a point-like source. The $\theta_{1}$ and $\theta_{2}$ values are calculated from the Monte Carlo simulations searching for the most significant difference between a purely point source and a source with an extended emission for a given size and profile. For each considered flux, radius and profile of the extended emission we calculated the value of $f$ and the corresponding significance of the extension with MC simulations. To minimize the systematic errors in modeling a point source we selected a data sample taken from the Crab Nebula (normalized to the Mrk 421 or Mrk 501 flux). The extension of the Crab Nebula in the VHE $\gamma$-rays, as shown in Aharonian et al. (2000), is $<0.025^{\circ}$, which makes it a point-like source for MAGIC. The mean spectral slopes of both Mrk 501 and Mrk 421 in the data sample are also rather similar to that of the Crab Nebula.

\section{Analysis of the systematic effects}

Systematic effects can degrade the precision of the estimation of the arrival direction of $\gamma$-rays. Some of those effects, if not properly taken into account, can emulate an extended emission around the point-like source. Here we present a study of such effects.

\subsection{Optical PSF}

The optical PSF of the telescope smears the images. This increases Width and Length of an image, thus changing its ellipticity. Therefore, the DISP method is affected and the $\theta^{2}$ distribution broadens. Variation of the optical PSF will be reflected in the measured angular resolution. The optical PSF varies across the field of view of the parabolic reflector of MAGIC. Varying gravitational loading of the mirror during observations produces small 

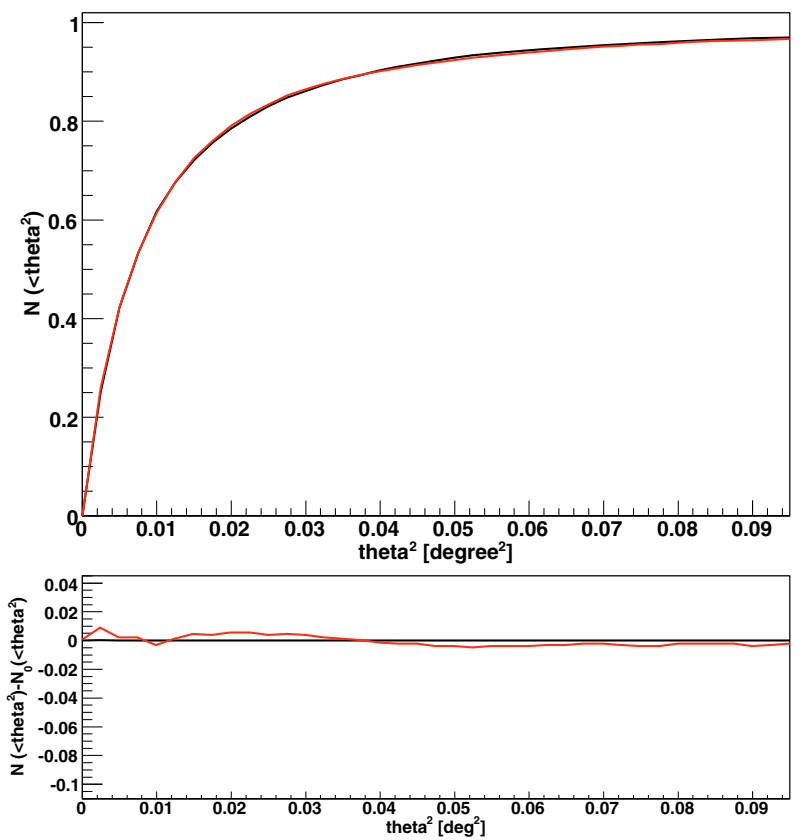

Fig. 3. Monte Carlo cumulative $\theta^{2}$ distributions for a point-like source for two different values of the optical PSF of the reflector: $\sigma=0.036^{\circ}$ (black), $\sigma=0.044^{\circ}$ (red) (upper panel), and a difference between them (lower panel). (This figure is available in color in electronic form).

deviations of the optical PSF that are corrected by the active mirror control system of MAGIC. The largest observed variations of the PSF of MAGIC are in the range of $20 \%$.

In Fig. 3 we show the result of a study of the influence of the optical PSF on the shape of the $\theta^{2}$ distribution. An increase of the optical PSF by e.g. $20 \%$ is equivalent to $\lesssim 2 \%$ admixture of an extended source with a characteristic extension radius of $0.2^{\circ}$. We thus conclude that this effect is negligible for our study.

\subsection{Spectral index}

As mentioned above, the $\theta^{2}$ estimation is more precise for higher energy $\gamma$-rays. Therefore, a source with a harder spectrum will have a more peaked $\theta^{2}$ distribution.

Figure 4 shows a comparison of cumulative $\theta^{2}$ distributions for spectral indices $-2.2,-2.4$ and -2.6 . The broadening of the $\theta^{2}$ distribution that corresponds to a steepening of the spectral index by 0.2 (a typical systematic error in spectral index determination) is comparable to having a $3 \%$ admixture of extended emission with a characteristic extension of $0.2^{\circ}$. Compared to the statistical uncertainties of this study, this effect is small.

\subsection{Mispointing}

The possible mispointing of the telescope limits the ability of IACTs to distinguish between point-like and extended sources. Using a strong source like the Crab Nebula or Mrk 421 one can estimate the source position and then calculate the mispointing as a difference between the true and the estimated positions.

As it is shown in Fig. 5 the accuracy of the source position reconstruction is $\lesssim 0.02-0.03^{\circ}$ for these observations. This agrees with our previous studies (Albert et al. 2008d). The value is well below the angular resolution of the telescope and the investigated extensions of the AGN halos.
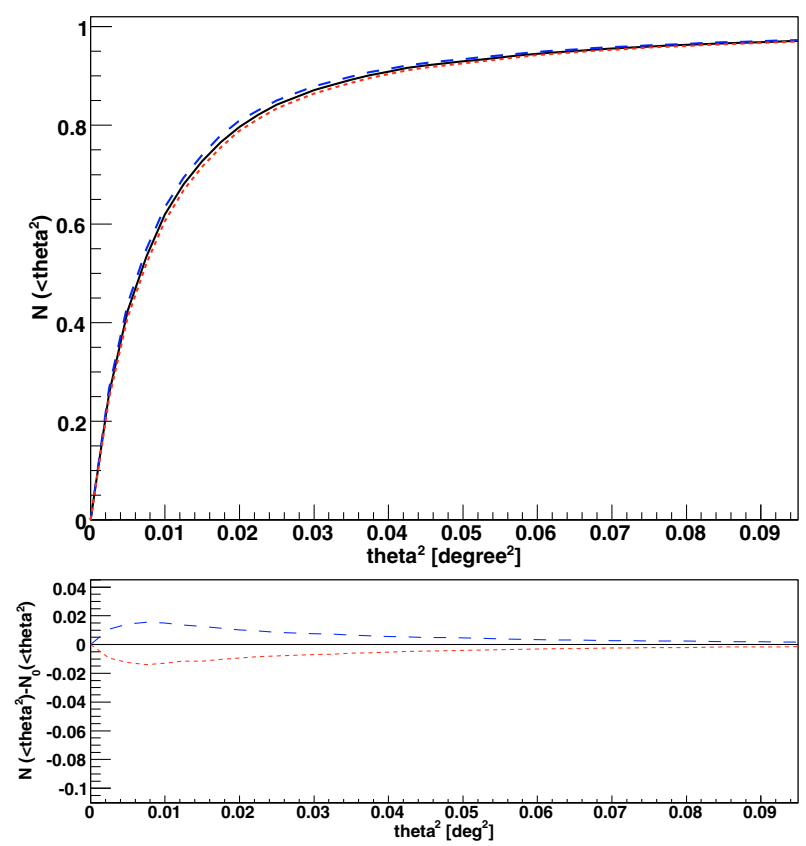

Fig. 4. Monte Carlo cumulative $\theta^{2}$ distributions for a point-like source with a different spectral index: -2.2 (blue dashed), -2.4 (black solid) -2.6 (red dotted) (upper panel), and a difference between them (lower panel). (This figure is available in color in electronic form).

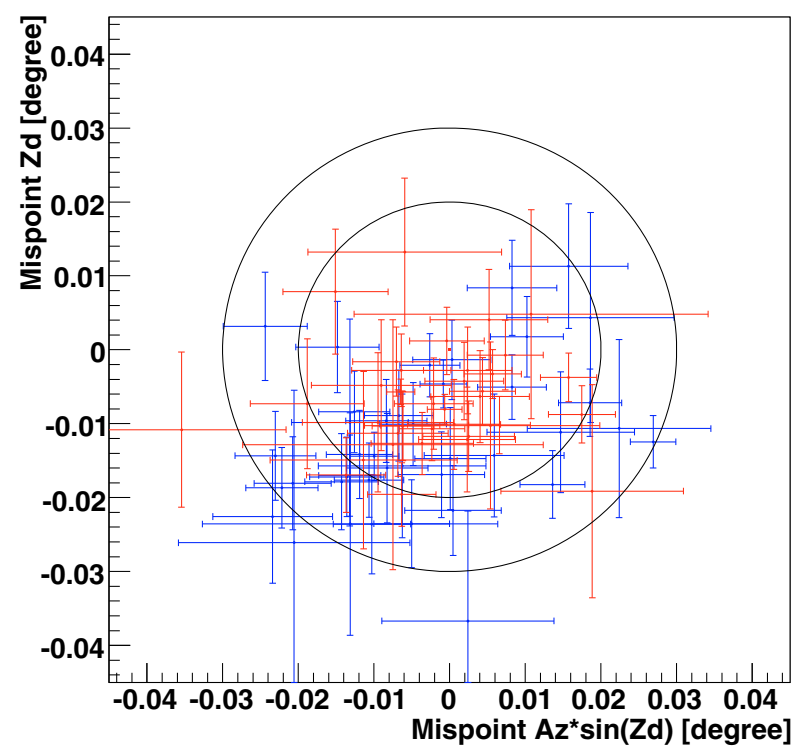

Fig. 5. Difference between the true and the estimated source positions for Mrk 421 (red) and Crab Nebula (blue). Circles with radii $0.02^{\circ}$ and $0.03^{\circ}$ show the characteristic mispointing scale. (This figure is available in color in electronic form).

\section{Observations}

MAGIC, consisting of two $17 \mathrm{~m}$ diameter telescopes, is located on the Canary island of La Palma at the Roque de los Muchachos Observatory (at 2200 m.a.s.l). The data presented in this paper has been taken with the first MAGIC telescope.

Mrk 421 and Mrk 501 are nearby blazars and well-known VHE $\gamma$-ray sources. Their spectra have been measured up to $\sim 10-20 \mathrm{TeV}$ (e.g. Aharonian et al. 1999b; Krennrich et al. 2001). At those energies absorption in EBL radiation fields is important so one may expect an extended emission component due to an AGN halo. Both sources are strongly variable. They have been monitored by various VHE $\gamma$-ray telescopes for 
nearly 20 years. and both quiescent states, with flux as low as $\sim 0.15$ C.U. (Crab unit), and giant flares with flux up to $\sim 10$ C.U., have been observed.

During 1995-1999 (with the exception of short time flares) the flux detected by the HEGRA instrument from Mrk 421 was well below 1 C.U. In 2000 it increased to $\sim 1$ C.U., and it further increased to $\sim 2.5$ C.U. in 2001 (Aharonian et al. 2003). The observed spectrum fits a power-law with a spectral index -2.4 and an exponential cut-off at the energy of $3.4 \mathrm{TeV}$. The MAGIC observations of Mrk 421 performed between November 2004 and April 2005 show that the flux varied between 0.5-2 C.U. The cut-off energy in this time period appeared to be lower, $\sim 1.4 \mathrm{TeV}$ (Albert et al. 2007a). In the low state the source had a steeper spectrum with a spectral index $\sim-3$ between 0.5 and $7 \mathrm{TeV}$ (Aharonian et al. 2002, 2003).

Observations of Mrk 501 are equally interesting. Historically the strongest activity period for this source was during 1997 . The mean flux observed by the HEGRA, the Whipple and the CAT telescopes in that year was 1.3-3 C.U. The same instruments observed Mrk 501 in 1998-1999 in a low state of $\sim 0.15$ C.U. The source became more active in 2000 , with a flux measured by the HEGRA and Whipple instruments of 0.35-1.2 C.U. (see review of all these observations in Albert et al. 2007b). MAGIC observations of Mrk 501 in 2005 yielded a mean flux of $\sim 0.5$ C.U. (Albert et al. 2007b), while observations performed in 2006 showed a low state at $\sim 0.2$ C.U. with a spectral index -2.8 (Anderhub et al. 2009). The spectra of Mrk 501 can be well described by a (possibly curved) power-law (Albert et al. 2007b).

For studing the possible extended VHE $\gamma$-ray emission we selected recent MAGIC observations of Mrk 421 and Mrk 501. To minimize systematic errors, only data taken at low $\left(<30^{\circ}\right)$ zenith angles were used.

Data from Mrk 501 were taken in 2008 Apr. and 2008 May in the so-called ON/OFF mode (where the source is in the center of the camera). After quality cuts, $26 \mathrm{~h}$ of ON data were selected and $50 \mathrm{~h}$ of OFF data were used for the background estimate. Mrk 501 was in a rather low state during this interval (mean flux in the sample $\sim 15 \%$ C.U.). The entire spectrum can be well fitted with a single power law with a spectral index of $-2.42 \pm$ $0.03_{\text {stat }} \pm 0.2_{\text {syst }}$ After correcting for the absorption due to the EBL, using the Franceschini et al. (2008) model, we obtain the source spectrum with an index of $-2.24 \pm 0.03_{\text {stat }} \pm 0.2_{\text {syst }}$.

The Mrk 421 data were obtained during 2007 Dec.2009 Feb. After quality, cuts $38 \mathrm{~h}$ of data were selected. The data was taken in the so called wobble mode, where the source position was shifted $0.4^{\circ}$ from the center of the camera. The opposite (with respect to the camera center) position was used for estimating the background. Since the background data were obtained simultaneously with the source data, the systematic errors are small. A disadvantage of this approach is that if the source extension is as large as $\sim 0.4^{\circ}$, the signal and the background regions overlap. This effect, which can reduce the sensitivity, has been studied and included in the Monte Carlo simulations. In the analyzed data sample Mrk 421 was in a high state ( 1.3 C.U.). Its spectrum in the broad energy range is best fitted with a flat (spectral index -2) power law with an exponential break point at $E_{\text {cut }}=2.1 \mathrm{TeV}$ ). In the limitted energy range $0.3-3 \mathrm{TeV}$ the spectrum can be fit by a power law with a spectral index of $-2.42 \pm 0.02_{\text {stat }} \pm 0.2_{\text {syst }}$.

The $\theta^{2}$ distribution for a point like source was calculated using the Crab Nebula data. We used $43 \mathrm{~h}$ of wobble mode Crab Nebula data, taken between 2007 Oct. and 2009 Mar., and $17 \mathrm{~h}$ of ON mode data taken during 2007 Dec. and 2008 Jan. This
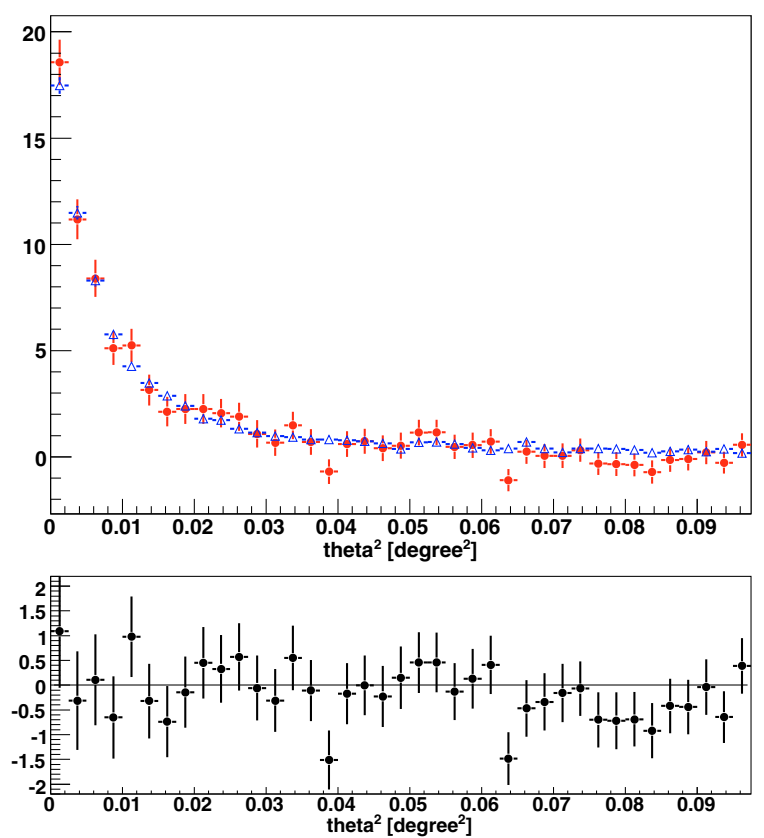

Fig. 6. Comparison of the excess $\theta^{2}$ distribution for $26 \mathrm{~h}$ of data from Mrk 501 (red circles) and a point-like source (blue triangles, 17h of Crab Nebula data) (upper panel) and the difference of both distributions (lower panel). (This figure is available in color in electronic form).
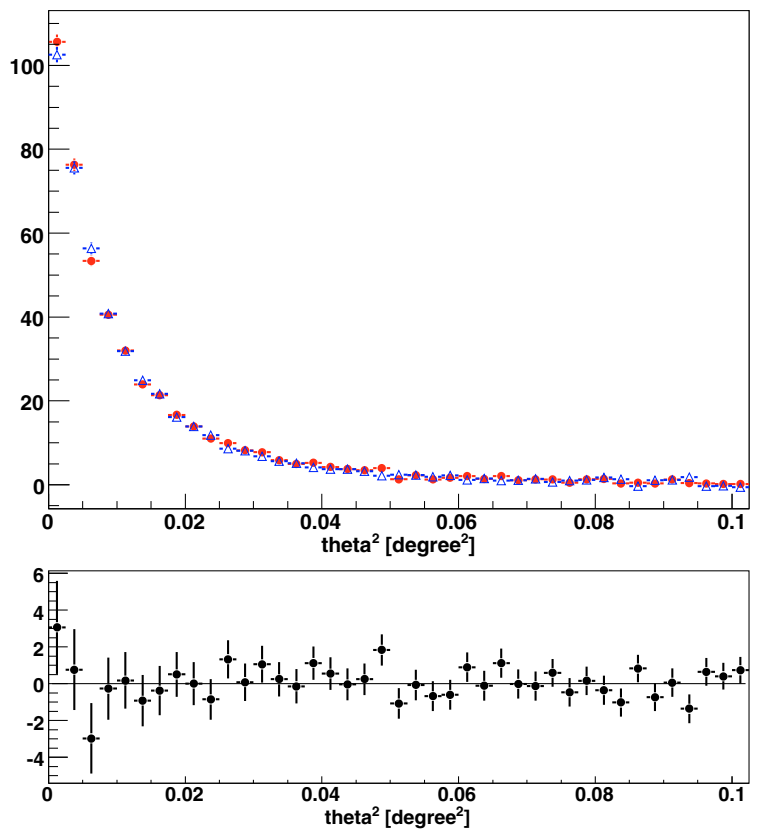

Fig. 7. Comparison of the excess $\theta^{2}$ distribution for $38 \mathrm{~h}$ of data from Mrk 421 (red circles) and a point-like source (blue triangles, $43 \mathrm{~h}$ of Crab Nebula data) (upper panel) and the difference of both distributions (lower panel). (This figure is available in color in electronic form).

data-set can be represented by a power law with a spectral index of $-2.3 \pm 0.02_{\text {stat }} \pm 0.2_{\text {syst }}$ for the energy band $0.3-3 \mathrm{TeV}$.

\section{Results}

$\theta^{2}$ distributions obtained for Mrk 501 and Mrk 421 are presented in Figs. 6 and 7.

For both sources the $\theta^{2}$ distributions match a point-like distribution. Using the first 12 bins, which contain most of the excess events, we calculate $\chi^{2} / n_{\text {d.o.f. }}=7.0 / 11$ (for Mrk 421) and 3.8/11 (for Mrk 501). 

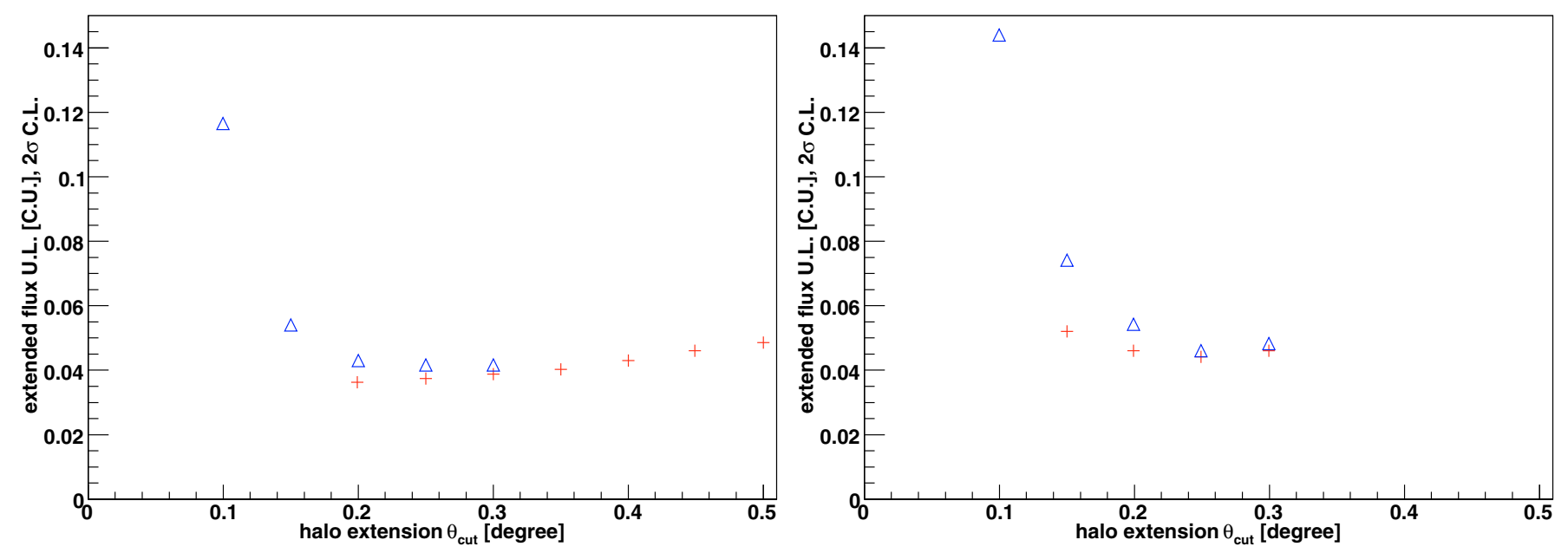

Fig. 8. Upper limit on the flux $(E>300 \mathrm{GeV})$ of the extended emission from Mrk 501 (left figure) and Mrk 421 (right figure) in the C.U. for different source profiles and extensions $\mathrm{d} N / \mathrm{d} \theta \propto \theta^{\beta}: 0^{\circ}<\theta<\theta_{\text {cut }}$ : flat disk $(\beta=1)$ (blue triangles); $0.1^{\circ}<\theta<\theta_{\text {cut }}: \beta=-1$ (red crosses). See text for more details. (This figure is available in color in electronic form).

The sensitivity for the detection of an extended emission depends on the extension size and profile. In our calculations we assumed a power law angular profile for the emission $(\mathrm{d} N / \mathrm{d} \theta \propto$ $\left.\theta^{\beta}\right)$ with various steepness indices $\beta=1,0,-1,-2$. We performed the calculations for extended emission radii up to $0.5^{\circ}$ for Mrk 501. For Mrk 421, the data were taken in wobble mode which allowed the estimate of the background from the same data set. We calculated the upper limits for the extension radii up to $0.3^{\circ}$. In this case there is no overlap between the signal and the background regions in the wobble mode observations. In Fig. 8 we present upper limits on the flux from the extended emission calculated for different extension radii and profiles for Mrk 501 (left figure) and Mrk 421 (right).

Flux upper limits for an extended emission from Mrk 501 for different energy thresholds are shown in Fig. 9. the upper limit for the extension radius $\sim 0.2^{\circ}$ ranges from $\sim 4 \%$ C.U. above $300 \mathrm{GeV}$ to $6,7 \%$ C.U. above $1 \mathrm{TeV}$, depending on the source profile.

\section{Discussion}

As can be seen in Fig. 8 the most stringent upper limits for the halo search for $E_{\gamma} \simeq 300 \mathrm{GeV}$ are achieved for a source extension of $0.2^{\circ}-0.25^{\circ}$ for Mrk 501 ( $4 \%$ C.U.) and $0.2^{\circ}-0.3^{\circ}$ for Mrk 421 (5\% C.U.). We checked that the upper limits for the emission profile $\mathrm{d} N / \mathrm{d} \theta \propto \theta^{\beta}, 0.1^{\circ}<\theta<\theta_{\text {cut }}, \beta=-2$ or $\beta=0$ are nearly the same as for the case of $\beta=-1$. For both sources, the best upper limits on the extended source flux are about $30 \%$ of the quiescent point source flux. It is also interesting to note that for an extension size $>0.2^{\circ}$ there is only a marginal dependence of the upper limits on the emission profile. Extension sizes $<0.2^{\circ}$, becoming comparable to the telescope's PSF, provide worse upper limits.

The non-detection of extended emission around Mrk 501 and Mrk 421 in the $0.3-1 \mathrm{TeV}$ energy range imposes restrictions on the properties of the highest-energy $\gamma$-ray emission from these sources and on the physical characteristics of the intergalactic medium around the sources. Cascade photons with an energy of $E_{\gamma} \simeq 300 \mathrm{GeV}$ result from absorption of the primary $\gamma$-rays in the energy range $E_{\gamma_{0}} \simeq 20\left[E_{\gamma} / 0.3 \mathrm{TeV}\right]^{1 / 2} \mathrm{TeV}$ that propagate over the distance $D_{\gamma} \simeq 40 \kappa\left[E_{\gamma_{0}} / 20 \mathrm{TeV}\right] \mathrm{Mpc}$ (Neronov \& Semikoz 2009). Here $\kappa \sim 1$ is a numerical factor that accounts for the uncertainty of the EBL models, which give $0.6<\kappa<2.5$ for Kneiske et al. (2004); Stecker et al. (2006); Primack et al. (2009); Franceschini et al. (2008).

Assuming that the EGMF strength is much higher than $B$ $10^{-12} \mathrm{G}$, as in the halo model of Aharonian et al. (1994), the derived constraint on the extended source fluxes can be used to constrain the isotropic primary source power at energies above $\simeq 20 \mathrm{TeV}$. This is especially interesting in the view of the recent discovery of $\mathrm{TeV} \gamma$-ray emission from the nearby radio galaxies (Aharonian et al. 2006; Albert et al. 2008b; Acciari et al. 2009; Aharonian et al. 2009). Within the general AGN unification scheme (Urry et al. 1991), the high energy peaked BL Lacs (HBL), such as Mrk 421 and Mrk 501, are believed to be aligned relativistically beamed FR I type radio galaxies, such as M 87 and Cen A. According to the AGN unification scheme, the detection of $\mathrm{TeV} \gamma$-ray emission from M87 and Cen A indicates that HBLs produce both beamed and isotropic $\mathrm{TeV}$ emission. The existence of such an isotropic component of VHE $\gamma$-ray emission from Mrk 421 and/or Mrk 501 is difficult to verify because the isotropic emission would produce a much smaller contribution to the $0.1-1 \mathrm{TeV}$ band point source emission. However, if the isotropic emission spectrum of Mrk 421 and/or Mrk 501 extends, similarly to M87 (Aharonian et al. 2006), to energies $E_{\gamma_{0}} \geq 20 \mathrm{TeV}$, absorption of the isotropically emitted $\gamma$-rays on the EBL leads to the production of an extended emission halo around the HBL. The detection of an extended halo, as discussed by Aharonian et al. (1994), would provide direct evidence for the existence of an isotropic multi-TeV emission from HBLs.

To constrain the isotropic emission from Mrk 501 and Mrk 421 from the limits on the extended emission flux requires an estimate of the fraction of the halo flux within the measurement region of the radius $0.1^{\circ}-0.5^{\circ}$. The observable angular size of the halos around Mrk 421 and Mrk 501 (both at the redshifts $z \simeq 0.03$ and distance $D \sim 150 \mathrm{Mpc}$ ) at the energy $E_{\gamma} \simeq 300 \mathrm{GeV}$ is expected to be $\Theta \sim D_{\gamma} / D \simeq 15^{\circ} \mathrm{K}$. Assuming a surface brightness profile $\mathrm{d} N_{\gamma} / \mathrm{d} \Omega \sim 1 / \theta$ like in Aharonian et al. (1994), one finds that the region $\theta \leq 0.5^{\circ}$ around the source contains $\sim 3 \%$ of the halo emission. The halo is expected to be more compact at $1 \mathrm{TeV}, \Theta \simeq 8.3^{\circ} \mathrm{K}$, so the central $\theta<0.5^{\circ}$ region contains $\sim 6 \%$ of the halo flux. This means that the total isotropic luminosity of Mrk 421 and Mrk 501 must be less than $L_{\text {halo }}\left(E_{\gamma_{0}}>20 \mathrm{TeV}\right) \simeq 1.3 \kappa L_{\mathrm{Crab}}(E>0.3 \mathrm{TeV})$ and $L_{\text {halo }}\left(E_{\gamma_{0}}>36 \mathrm{TeV}\right) \simeq 1.3 \kappa L_{\mathrm{Crab}}(E>1 \mathrm{TeV})$. This limit is not 


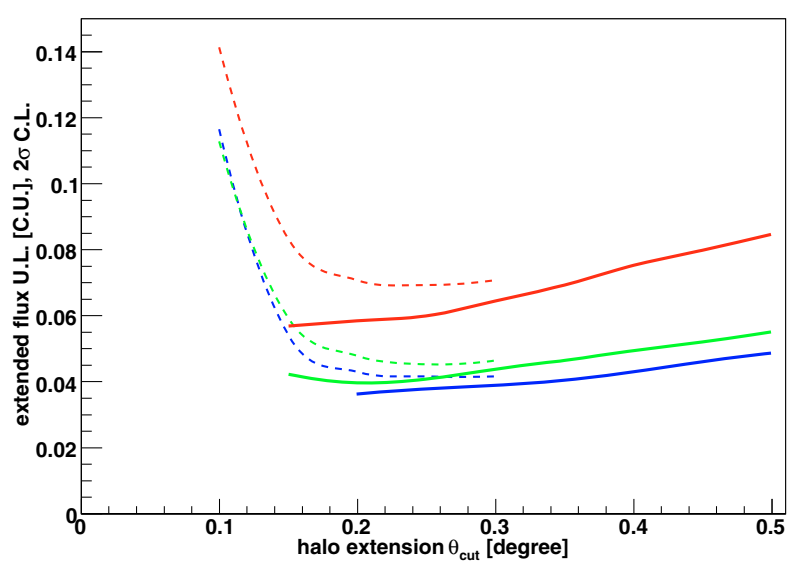

Fig. 9. Upper limit (in C.U.) on the flux of the extended emission from Mrk 501 for different energy thresholds, different source profiles and extensions $\mathrm{d} N / \mathrm{d} \theta \propto \theta^{\beta}: 0^{\circ}<\theta<\theta_{\text {cut }}$ : flat $(\beta=1)$ (dashed lines), $0.1^{\circ}<\theta<\theta_{\text {cut }}, \beta=-1$ (solid lines). $E_{\text {th }}=1000 \mathrm{GeV}$ (red), $600 \mathrm{GeV}$ (green) and $300 \mathrm{GeV}$ (blue). (This figure is available in color in electronic form).

very restrictive. Our analysis shows that the isotropic luminosity of HBLs can be more efficiently constrained by the search for an extended emission from a more distant source (for which the angular size of extended halos is smaller) and/or with observations from wider field-of-view instruments.

If the EGMF is much weaker than $10^{-12} \mathrm{G}$ the deflections of cascade $\mathrm{e}^{+} \mathrm{e}^{-}$pairs are not large enough to isotropize cascade $\gamma$-ray emission and no isotropically emitting halo is formed around the point source. The extended cascade source should appear more compact depending on the EGMF strength. Nondetection of extended emission constrains possible range of EGMF strength. In this short discussion we mention only limits on the EGMF with large correlation length. These limits could be extended to the case of arbitrary correlation length of the EGMF in a straightforward way, using the formalism of Neronov \& Semikoz (2009). The absence of extended emission at $E_{\gamma} \sim$ $300 \mathrm{GeV}$ imposes a bound on the EGMF only when the timeaveraged primary (beamed) source emission spectrum extends to energies above $E_{\gamma_{0}} \geq 20 \mathrm{TeV}$ (see above). Unfortunately, the observations in the $\mathrm{TeV}$ band and extreme variability of TeV-emitting blazars do not permit deriving the time averaged spectra of the sources.

For Mrk 421, a high energy cut-off in the spectrum at $E_{\text {cut }} \simeq$ 1-5 TeV has been repeatedly reported (Krennrich et al. 2001) (see however, Konopelko et al. 2008). If such a cut-off is intrinsic and present in the time-averaged spectrum, the source luminosity at $20 \mathrm{TeV}$ is expected to be a factor of $\geq 15$ lower than the luminosity at $300 \mathrm{GeV}$ (assuming an intrinsic power law photon index of $\Gamma \simeq-1.7$, close to that measured by Fermi Abdo et al. 2009). In this case the flux of the extended cascade emission at the energies $\geq 300 \mathrm{GeV}$ is a factor $\geq 15$ lower than the point source flux. This is consistent with the upper limits derived above. In the scenario of intrinsic cut-off in the Mrk 421 energy spectrum, the upper bound on extended emission around Mrk 421 derived from MAGIC observations does not constrain the strength of the EGMF.

On the contrary, the time-averaged spectrum of Mrk 501 probably extends to much higher-energies. No intrinsic high energy cut-offs in the low or high activity state of the source have been reported in the literature. This implies that the source luminosity at the energies above $20 \mathrm{TeV}$ is of the same order as the luminosity at $300 \mathrm{GeV}$. The bound on the extended source flux, $\sim 0.04$ C.U. at $300 \mathrm{GeV}$, is a factor $\simeq 4$ lower than the point source flux at the same energy. If the intrinsic source flux at $\sim 20 \mathrm{TeV}$ energy is higher than $1 / 4$ of the flux at $300 \mathrm{GeV}$, the bound on the extended source flux, derived from MAGIC observations, can impose constraints on the strength of the EGMF within the region $D \sim D_{\gamma} \sim 40 \kappa \mathrm{Mpc}$ around the blazar. The spectrum of Mrk 501 from the analyzed data sample after correction for the absorption using the Franceschini et al. (2008) model (which provides a relatively low level of absorption) has a rather hard spectral index of -2.24 . This yields only a factor $(20 \mathrm{TeV} / 0.3 \mathrm{TeV})^{0.24}=2.7$ decrease in the SED from the energy of 0.3 to $20 \mathrm{TeV}$. However, both the uncertainties of the EBL absorption models and the fact that Mrk 501 is known to be variable, tells us that the time-averaged spectrum over a longer periods of time may be softer than that in the data sample we have used. Measurement of the intrinsic time-averaged source flux at $20 \mathrm{TeV}$ will be possible only after precise measurements of the EBL in the mid-infrared and regular monitoring of the source over years. Therefore, we give below only a rough estimate of the range of magnetic fields that might be constrained with the Mrk 501 data.

Assuming that the correlation length of the EGMF is much larger than the inverse Compton energy loss distance of electrons with energies $E_{\mathrm{e}} \sim E_{\gamma_{0}} / 2 \sim$ $10 \mathrm{TeV}, D_{\mathrm{e}} \simeq 30\left[E_{\mathrm{e}} / 10 \mathrm{TeV}\right]^{-1} \mathrm{kpc}$, the size of the extended source around Mrk 501 is expected to be $\Theta_{\text {ext }} \simeq$ $0.4^{\circ}[\tau / 3.5]^{-1}\left[E_{\gamma} / 300 \mathrm{GeV}\right]^{-1}\left[B / 10^{-14} \mathrm{G}\right]$ where $\tau=D / D_{\gamma}$ is the optical depth for the primary $\gamma$-rays with respect to the pair production on the EBL (Neronov \& Semikoz 2009). The nondetection of an extended source with $0.15^{\circ}<\Theta_{\text {ext }}<0.5^{\circ}$ at an energy $300 \mathrm{GeV}$ might constrain EGMF with a strength in the range $4 \times 10^{-15}<B<1.3 \times 10^{-14} \mathrm{G}$. A significant secondary cascade emission at $1 \mathrm{TeV}$ is possible if there is no cut-off in the primary spectrum below $40 \mathrm{TeV}$. In this case non-detection of the extended emission at $1 \mathrm{TeV}$ can be used for additionally excluding EGMF strengths up to $B \simeq 8 \times 10^{-14} \mathrm{G}$.

An efficient way to enhance the sensitivity of this search will be to use much longer observations, if the systematics (e.g. the spot size, pointing accuracy) are well under control. For example, if one would use $400 \mathrm{~h}$ of on-source time (during 1 year, acceptable zenith angles) then a simple scaling as a square root of time will provide a sensitivity on the level of $1 \%$ C.U.

Better sensitivity for a halo search could also be achieved if the source is observed in a low emission state. In the high emission state the extended component remains constant, while the point-like emission provides additional background, hence degrading the sensitivity. This effect is especially important for sources that may have an extension comparable to the angular resolution of the telescope. Another improvement in sensitivity comes from increased angular resolution of the instrument, especially when the emission radius is small. This would help to disentangle the extended component for the primary, pointlike component down to smaller extensions. For example, the planned CTA-like telescope array are intended to achieve an angular resolution of about 2 arcmin (Hillas 1989) permitting the probing of a halo extension three times smaller than found for the current study. By measuring with an instrument one order of magnitude more sensitive than MAGIC, e.g. CTA, it seems realistic to expect a sensitivity of $\sim 0.1 \%$ Crab for the halo search for sources with an extension $>0.1-0.2^{\circ}$. An observation time of a few hundred hours will be necessary for such a study. 


\section{Conclusions}

We have developed a novel method based on the Random Forest algorithm for the estimation of the source position. This method improves the angular resolution by $\sim 20-30 \%$.

Using 26h of observations for Mrk 501 and 38h for Mrk 421, no extended emission has been detected around these sources. Our study shows that if there is an extended emission around Mrk 501, its flux is $<4 \%$ C.U. (see Fig. 8 left), for an analysis energy threshold of $300 \mathrm{GeV}$. For Mrk 421 the upper limits are less stringent because the source was in a high emission state that causes the tail of the point-like emission to extend into the halo region thus creating additional background. The constraint on the extended emission flux from Mrk 421 is $<5 \%$ C.U. (see Fig. 8 right). We analyzed different systematic errors connected with the observations of extended sources. We find that they produce a neglibile contribution compared to the upper limits obtained on the flux of the extended emission component of Mrk 421 and Mrk 501.

With the second telescope, the MAGIC system can be operated in the stereo mode. By combining simultaneous information from both telescopes, the angular resolution will be improved. This will allow us to perform a search for the AGN halo with a higher sensitivity. The stereo observations improve the sensitivity by 2.5 times at lower energies $(\sim 100 \mathrm{GeV})$. Moreover, the better signal-to-noise ratio reduces the possible bias due to systematic errors. It will allow us to perform a sensitive search for halos at lower energies, thus extending the range of the EGMF strengths investigated with this method.

A future $\gamma$-ray telescope project - the Cherenkov Telescope Array is aiming for a much better angular resolution. In this case lower halo extensions can be investigated with an improved sensitivity.

Acknowledgements. We would like to thank the Instituto de Astrofisica de Canarias for the excellent working conditions at the Observatorio del Roque de los Muchachos in La Palma. The support of the German BMBF and MPG, the Italian INFN, the Swiss National Fund SNF, and the Spanish MICINN is gratefully acknowledged. This work was also supported by the Polish MNiSzW Grant No. N203 390834, by the YIP of the Helmholtz Gemeinschaft, and by grant DO02-353 of the the Bulgarian National Science Fund.

\section{References}

Abdo, A. A., Ackermann, M., Ajello, M., et al. 2009, ApJ, 707, 1310

Acciari, V. A., Aliu, E., Arlen, T., et al. 2009, Science, 325, 444

Aharonian, F. A., Coppi, P. S., \& Völk, H. J. 1994, ApJ, 423, L5

Aharonian, F. A., Akhperjanian, A. G., Barrio, J. A., et al. 1999a, A\&A, 342, 69

Aharonian, F. A., Akhperjanian, A. G., Barrio, J. A., et al. 1999b, A\&A, 349, 11

Aharonian, F. A., Akhperjanian, A. G., Barrio, J. A., et al. 2000, A\&A, 361, 1073

Aharonian, F. A., Akhperjanian, A. G., Barrio, J. A., et al. 2001a, A\&A, 366, 746

Aharonian, F., Akhperjanian, A., Barrio, J., et al. 2001b, ApJ, 546, 898

Aharonian, F., Akhperjanian, A., Beilicke, M., et al. 2002, A\&A, 393, 89

Aharonian, F., Akhperjanian, A., Beilicke, M., et al. 2003, A\&A, 410, 813

Aharonian, F., Akhperjanian, A. G., Bazer-Bachi, A. R., et al. 2006, Science, 314,1424

Aharonian, F., Akhperjanian, A. G., Bazer-Bachi, A. R., et al. 2007, ApJ, 664, L71

Aharonian, F., Akhperjanian, A. G., Anton, G., de et al. 2009, ApJ, 695, L40

Albert, J., Aliu, E., Anderhub, H., et al. 2007a, ApJ, 663, 125

Albert, J., Aliu, E., Anderhub, H., et al., 2007b, ApJ, 669, 862

Albert, J., Aliu, E., Anderhub, H., et al., 2008a, Phys. Lett. B, 668, 253

Albert, J., Aliu, E., Anderhub, H., et al. 2008b, ApJ, 685, L23

Albert, J., Aliu, E., Anderhub, H., et al. 2008c, NIM A, 588, 424

Albert, J., Aliu, E., Anderhub, H., et al. 2008d, ApJ, 674, 1037

de Angelis, A., Persic, M., \& Roncadelli, M. 2008, Modern Phys. Lett. A, 23, 315
Aliu, E., Anderhub, H., Antonelli, L. A., et al. 2009, Astropart. Phys., 30, 293 Anderhub, H., Antonelli, L. A., Antoranz, P., et al. 2009, ApJ, 705, 1624 Beck, R. 2008, AIP Conf. Proc., 1085, 83

Bock, R. K., Chilingarian, A., Gaug, M., et al. 2004, NIM A, 516, 511

Carilli, C. L., \& Taylor, G. B. 2002, ARA\&A, 40, 319

Dai, Z. G., Zhang, B., Gou, L. J., Mészáros, P., \& Waxman, E. 2002, ApJ, 580, L7

Dolag, K., Kachelriess, M., Ostapchenko, S., \& Tomas, R. 2009, ApJ, 703, 1078 Domingo-Santamaria, E., Flix, J., Scalzotto, V., Wittek, W., \& Rico, J. 2005, Proc. 29th ICRC, Pune, 101

Elyiv, A., Neronov, A., \& Semikoz, D. 2009, Phys. Rev. D, 80, 023010

Finke, J. D., Razzaque, S., \& Dermer, C. D. 2010, ApJ, 712, 238

Franceschini, A., Rodighiero, G., \& Vaccari, M. 2008, A\&A, 487, 837

Gilmore, R. C., Madau, P., Primack, J. R., \& Somerville, R. S. 2008, AIP Conf.

Proc., 1085, 577

Grasso, D., \& Rubinstein, H. R. 2001, Phys. Rept. 348, 163

Hillas, A. M. 1985, Proc. 19th ICRC, La Jolla 3, 445

Hillas, A. M. 1989, Very High Energy Gamma Ray Astronomy, Crimea, 134

Kneiske, T. M., Bretz, T., Mannheim, K., \& Hartmann, D. H. 2004, A\&A, 413, 807

Konopelko, A., Cui, W., Duke, C., \& Finley, J. P. 2008, ApJ, 679, L13

Krennrich, F., Badran, H. M., Bond, I. H., et al. 2001, ApJ, 560, L45

Kronberg, P. P. 1994, Rept. Prog. Phys. 57, 325

Kronberg, P. P., Kothes, R., Salter, C. J., \& Perillat, P. 2007, ApJ, 659, 267

Kulsrud, R. M., \& Zweibel, E. G. 2008, Rept Prog. Phys., 71, 046901

Mazin, D., \& Raue, M. 2007, A\&A, 471, 439

Murase, K., Takahashi, K., Inoue, S., Ichiki K., \& Nagataki, S. 2008, ApJ, 686, L67

Neronov, A., \& Semikoz, D. V. 2007, JETP Lett., 85, 579

Neronov, A., \& Semikoz, D. V. 2009, Phys. Rev. D, 80, 123012

Plaga, R. 1995, Nature 374, 430

Primack, J. R., Gilmore, R. C., \& Somerville, R. S. 2009, AIP Conf. Proc., 1085, 71

Stecker, F. W., de Jager, O. C., \& Salamon, M. H. 1992, ApJ, 390, L49

Stecker, F. W., Malkan M. A., \& Scully, S. T. 2006, ApJ, 648, 774

Urry, C. M., Padovani, P., \& Stickel, M. 1991, ApJ, 382, 501

Widrow, L. M. 2002, Rev. Mod. Phys., 74, 775

Xu, Y., Kronberg, P. P., Habib, S., \& Dufton, Q. W. 2006, ApJ, 637, 19

1 IFAE, Edifici Cn., Campus UAB, 08193 Bellaterra, Spain

2 INAF National Institute for Astrophysics, 00136 Rome, Italy

3 Università di Siena, and INFN Pisa, 53100 Siena, Italy

4 Technische Universität Dortmund, 44221 Dortmund, Germany

5 Universitat Autònoma de Barcelona, 08193 Bellaterra, Spain

6 Universidad Complutense, 28040 Madrid, Spain

7 Università di Padova and INFN, 35131 Padova, Italy

8 Inst. de Astrofísica de Canarias, 38200 La Laguna, Tenerife, Spain

9 University of Łódź, 90236 Lodz, Poland

10 Tuorla Observatory, University of Turku, 21500 Piikkiö, Finland

11 Deutsches Elektronen-Synchrotron (DESY), 15738 Zeuthen, Germany

12 ETH Zurich, 8093 Switzerland

13 Max-Planck-Institut für Physik, 80805 München, Germany

14 Universitat de Barcelona (ICC/IEEC), 08028 Barcelona, Spain

15 Universität Würzburg, 97074 Würzburg, Germany

16 Depto. de Astrofisica, Universidad, 38206 La Laguna, Tenerife, Spain

17 Università di Udine, and INFN Trieste, 33100 Udine, Italy

18 Institut de Ciències de l'Espai (IEEC-CSIC), 08193 Bellaterra, Spain

19 Inst. de Astrofísica de Andalucía (CSIC), 18080 Granada, Spain

20 Croatian MAGIC Consortium, Institute R. Boskovic, University of Rijeka, University of Split, 10000 Zagreb, Croatia

21 University of California, Davis, 95616-8677, USA

22 Inst. for Nucl. Research and Nucl. Energy, 1784 Sofia, Bulgaria

23 INAF/Osservatorio Astronomico and INFN, 34143 Trieste, Italy

24 ICREA, 08010 Barcelona, Spain

25 Università di Pisa, and INFN Pisa, 56126 Pisa, Italy

26 SDC Data Center for Astrophysics, Geneva Observatory, Chemin d'Écogia 16, 1290 Versoix, Switzerland

27 APC, 10 rue Alice Domon et Leonie Duquet, 75205 Paris Cedex 13, France 